\title{
Prevalence and Risk Factor of Diabetic Foot Ulcers in a Regional Hospital, Eastern Indonesia
}

\author{
Saldy Yusuf1,2, Mayumi Okuwa1, Muhammad Irwan'2, Saipullah Rassa'2, Baharia Laitung2, \\ Abdul Thalib', Sukmawati Kasim², Hiromi Sanada3 ${ }^{3}$, Toshio Nakatani' ${ }^{1}$, Junko Sugama 1,4* \\ ${ }^{1}$ Division of Health Sciences, Graduate School of Kanazawa University, Kanazawa, Japan \\ ${ }^{2}$ Wound Care Clinic, Griya Afiat, Makassar, Indonesia \\ ${ }^{3}$ Department of Gerontological Nursing/Wound Care Management, Faculty of Medicine, University of Tokyo, \\ Tokyo, Japan \\ ${ }^{4}$ Wellness Promotion Science Center, Institute of Medical, Pharmaceutical and Health Sciences, Kanazawa \\ University, Kanazawa, Japan \\ Email: "junkosgm@mhs.mp.kanazawa-u.ac.jp
}

Received 29 October 2015; accepted 15 January 2016; published 18 January 2016

Copyright (C) 2016 by authors and Scientific Research Publishing Inc.

This work is licensed under the Creative Commons Attribution International License (CC BY).

http://creativecommons.org/licenses/by/4.0/

(c) (i) Open Access

\begin{abstract}
Indonesia is one of the top ten diabetes mellittus (DM) countries. However as the main complication of DM, there was lack of studies related to diabetic foot ulcer (DFU). Thus, the aim of this study was to survey the prevalence of DFU risk factors and DFU among type 2 diabetes mellitus (T2DM) patients. An epidemiological study was conducted at an outpatient endocrine clinic in a regional hospital, eastern Indonesia. All T2DM participants attending research setting that were $\geq$ 18 years were included. Demographic and foot care behavior were assessed using minimum data sheet (MDS). Meanwhile, presence of risk factors was evaluated by using 5.07/10 g SemmesWeinsten Monofilament (SWM) for neuropathy and presence of angiopathy was evaluated with Ankle Brachial Index (ABI) by using a hand held Doppler (Bidop ES-100V3, Hadeco-Kawasaki, Japan) both dorsal and posterior tibialis foot. At the end of study, 249 T2DM participants were enrolled. The prevalence of DFU risk factors was 55.4\% (95\% CI: 53.7\% - 57.0\%), and prevalence of DFU was $12 \%$ (95\% CI: $10.3 \%$ - 13.6\%). Based on a logistic regression, predictors for DFU risk factors included age (OR: 1.04; 95\% CI: 1.005 - 1.074) and daily foot inspection (OR: 0.36; 95\% CI: 0.186 - 0.703). Meanwhile, the predictors for presence of DFU were insulin (OR: 9.37; 95\% CI: 2.240 - 39.182), shoes (OR: 0.05; 95\% CI: 0.007 - 0.294), spiritual belief that DM was a disease (OR: 0.04; 95\% CI: $0.004-0.326$ ) and belief that DM was a temptation from God (OR: 0.13; 95\% CI: $0.027-0.598)$. In conclusion, we recommend to educate high risk patients to understand positive foot care behavior as essentially preventive strategies to prevent presence risk and DFU.
\end{abstract}

${ }^{*}$ Corresponding author.

How to cite this paper: Yusuf, S., Okuwa, M., Irwan, M., Rassa, S., Laitung, B., Thalib, A., Kasim, S., Sanada, H., Nakatani, T. and Sugama, J. (2016) Prevalence and Risk Factor of Diabetic Foot Ulcers in a Regional Hospital, Eastern Indonesia. Open Journal of Nursing, 6, 1-10. http://dx.doi.org/10.4236/ojn.2016.61001 


\section{Keywords}

\section{Prevalence, Diabetes Complications, Risk Factors, Diabetic Foot Ulcers}

\section{Background}

Prevalence of Diabetes Mellitus (DM) in Indonesia is high. As one of the top ten DM countries [1] [2], prevalence of DM in Indonesia has been increasing from year to year. In 1983, prevalence of DM in Indonesia was $1.63 \%$ [3], increased $5.7 \%$ in 2007 [4] and is predicted to be $6.0 \%$ in 2030 [1] or was equal to 8.5 million in 2013 and will be 14.1 million patients in 2035 [2]. In addition, a national survey reported a high number of undiagnosed DM in Indonesia (4.3\%) [4] [5]. Therefore, prevalence of DM in Indonesia is potential to be higher than available data.

One of the major DM complications is the development of diabetic foot ulcer (DFU). International Working Group on Diabetic Foot (IWGDF) has proposed neuropathy and angiopathy as the main risk factors for development DFU [6]. Role of these risk factors has been explained biomechanically [7] and biologically [8]. In Western, neuropathy is determined by demography factors [9], while the development of DFU is mainly related to trauma, neuropathy and deformity [10]. However, most of the studies focused only on neuropathy or angiopathy.

Unlike in Western countries in Indonesia, there are only a few studies related to prevalence, associated factors for presence of risk and DFU. Previous study concludes that the main complications of DM in Indonesia are neuropathy (13\% - 78\%), microvascular complication (16\% - 53\%) and DFU (7.3\% - 24\%) [5]. However, there is inadequate information related to associated factors for presence of risk and DFU. In addition, the external data from Western country studies cannot be generalized into Indonesian setting since characteristics of demography, lifestyle and behavior are different. This fact leads to limitation of preventive strategies to prevent presence of risk and DFU based on Indonesian type 2 diabetes mellitus (T2DM) characteristics. Thus, objective of this epidemiology study is to evaluate prevalence, associated factors for presence of risk and DFU among T2DM patients in Makassar, eastern Indonesia.

\section{Methods}

\subsection{Setting and Participants}

Strengthening the reporting of observational studies in epidemiology (STROBE) guideline was used to design and report this epidemiological study [11]. Previous study reported that Ujung Pandang (now Makassar) was one of the most prevalent DM cities in Indonesia [12], including for asymptomatic DM [13]. Thus, research setting was conducted in outpatient endocrine clinic, Wahidin Sudirohusodo hospital, a regional hospital in Makassar (834 beds), which considered representative in eastern Indonesia.

Sample size was calculated by using power analysis equation [14], where P refers to the lifetime prevalence of DFU as 25\% [15]. Thus, our calculated sample size was 288 participants. Study populations were all T2DM patients who attended the research setting from May 2013 to February 2014 for DM therapy. Inclusion criteria were T2DM patients who have $\geq 18$ years old, this was our denominator and presence of DFU was numerator of study. T2DM patients who attended hospital other than endocrine outpatient clinic were excluded. Diagnosed T2DM patients are based on physician endocrine assessment and glycemic status according to American Diabetes Association (ADA) 2013 criteria [16], which are written in hospital medical records.

\subsection{Variables}

Dependent variables were presence of risk and DFU. Presence of risk was neuropathy or angiopathy as proposed by IWGDF [6]. Neuropathy was evaluated by using 5.07/10 g Semmes-Weinsten Monofilament (SWM) at four points of each foot (dorsal hallux, metatarsal I, III and V) [15], absence of one of the four sites is considered as neuropathy [17]. Meanwhile, presence of angiopathy was evaluated with Ankle Brachial Index (ABI) by using a hand held Doppler (Bidop ES-100V3, Hadeco-Kawasaki, Japan) both dorsal and posterior tibialis foot. An ABI $\leq$ 0.9 considered has peripheral ischemic [18]. Since there was inconsistent normal range of TBI, we reported as 
mean and standard deviation instead of categorical data [19]. DFU define as presence full thickness lesion on the skin as proposed by IWGDF [20].

Independent variables were demography, clinical foot problems and foot care behaviors. Participants' demographies were age, sex, occupation, religion, education, smoking status, DM therapy, duration of DM, body mass index (BMI), blood pressure and $\mathrm{HbA}_{1 \mathrm{C}}$ (at the time of visit or at least two months of previous data) was analyzed in hospital laboratories. Clinical foot problems were skin problems (dry skin, callus-corn-fissure and tinea pedis), nail problems (nail deformity and onychomycosis), foot deformity, ABI and Toe Brachial Index (TBI) status.

Foot care behaviors were interviewed by using questionnaire, including foot inspection, foot washing, nail trim, footwear inspection, and footwear practice. Participants' cultural and spiritual belief related to foot care behaviors was also interviewed by using open questions (do you have spiritual or cultural belief related to your foot care?). These items were developed based on clinical experience in Indonesian setting. To control bias, all of foot assessments, including determination of DFU were done by one investigator (Wound Care Nurse). All of assessments were written into paper-pencil form by co-authors and stored by primary investigator. Participants' anonimity was maintained by using identical number (ID number).

\subsection{Data Analysis}

Both ordinal and nominal data were described as absolute values and percentages (n, \%), while continuous data were reported as mean and standard deviation. Missing data were replaced with its group mean (11 TBI, 19 ABI, and $12 \mathrm{HbA}_{1 \mathrm{C}}$ data).

Univariate data were analyzed by $\chi^{2}$ test or Fisher exact test for categorical and Independent $t$ test for continuous data. Cut off $\mathrm{P}<0.1$ and logical reasons were used to select candidate of predictors into multivariate analysis. Possibilities for multicollinearity were diagnosed by reading variance inflation factor (VIF) by using regression linear. All of predictors with VIF $<5.0$ were entered into logistic regression with Forward LR methods. The significance level was set up at $95 \%$ with $\mathrm{P}=0.05$ (two tailed). All data were analyzed by SPSS version 16.0 software (SPSS, Inc. Chicago, IL).

\subsection{Ethical Consideration}

Ethical considerations were approved from Kanazawa University, Japan (Number: 438) and Hasanuddin University, Indonesia (Number:0866/H4.8.4.5.31/PP36-KOMETIK/2013). All of participants and family were received explanation and signed informed consent prior to data collection.

\section{Results}

\subsection{Prevalence of Risk and DFU}

There are 280 T2DM patients who have registered in the research setting, 259 participants agree to participate (response rate 92.5\%). At the end of the study, 10 participants are unable to complete assessment and remain 249 participants in the analysis. Participants without any risk are 90, with risk 112 (with neuropathy 14, neuropathy or deformity 64, and neuropathy or deformity or ischemic 34), history of DFU 14, history of amputation 3 and current DFU 30 of 249 participants. Overall, prevalence of presence of risk factors (excluding history and presence of DFU) is 55.4\% (95\% CI 53.7\% - 57.0\%) and prevalence for DFU (including history and presence of DFU) is $12.0 \%$ (95\% CI: $10.3 \%-13.6 \%)$.

\subsection{Univariate Analysis}

In univariate analysis we evaluate candidate of predictor for presence of risk and DFU based on demographic, clinical foot assessment and foot care bahaviors. With regard to demographic factors, age is older $(\mathrm{P}=0.021)$ for presence of risk, meanwhile religion ( $\mathrm{P}=0.032)$, education $(\mathrm{P}=0.023)$, DM therapy $(\mathrm{P}=0.020)$ and percentage of $\mathrm{HbA}_{1 \mathrm{C}}(\mathrm{P}=0.029)$ are associated with presence of DFU (Table 1). Based on clinical foot assessment, deformity $(\mathrm{P}=0.000)$ and $\mathrm{ABI}(\mathrm{P}=0.000)$ are associated with presence of risk factors. However, only dry skin has an evidence role in presence of DFU $(\mathrm{P}=0.001)$ (Table 2).

Related to participants' foot care behaviors, foot inspection has been less frequently at risk group, including 
Table 1. Univariate analysis of demography, general health and diabetes mellitus (DM) status for presence risk factors and diabetic foot ulcers (DFU).

\begin{tabular}{|c|c|c|c|c|c|c|c|c|c|c|c|c|c|c|}
\hline \multirow[b]{3}{*}{ Age (years) $\dagger$} & \multicolumn{7}{|c|}{ Presence Risk } & \multicolumn{7}{|c|}{ Diabetic Foot Ulcer (DFU) } \\
\hline & \multicolumn{2}{|c|}{$\begin{array}{c}\text { No Risk } \\
\mathrm{n}=90(\%)\end{array}$} & \multicolumn{2}{|c|}{$\begin{array}{c}\text { Presence Risk } \\
\text { n:112 (\%)* }\end{array}$} & \multicolumn{2}{|c|}{$\begin{array}{c}\text { Total } \\
\text { n:202 (\%) }\end{array}$} & \multirow{2}{*}{$\begin{array}{c}\mathrm{p} \\
0.021\end{array}$} & \multicolumn{2}{|c|}{$\begin{array}{c}\text { No DFU } \\
\text { n: } 219(\%)\end{array}$} & \multicolumn{2}{|c|}{$\begin{array}{c}\text { Presence } \\
\text { DFU } \\
\text { n: } 30(\%)\end{array}$} & \multicolumn{2}{|c|}{$\begin{array}{c}\text { Total } \\
\text { n: } 249(\%)\end{array}$} & \multirow{2}{*}{$\begin{array}{c}\mathrm{p} \\
0.143\end{array}$} \\
\hline & 59.7 & \pm 8.6 & 62.7 & \pm 9.2 & 61.3 & \pm 9.1 & & 61.54 & \pm 9.21 & 58.33 & \pm 11.20 & 61.15 & \pm 9.51 & \\
\hline Sex: Female & 56 & $(62.2)$ & 61 & $(54.5)$ & 117 & (57.9) & 0.316 & 125 & $(57.1)$ & 20 & $(66.7)$ & 145 & $(58.2)$ & 0.333 \\
\hline \multicolumn{15}{|l|}{ Occupation } \\
\hline House Wife & 31 & $(34.4)$ & 31 & $(27.7)$ & 62 & $(30.7)$ & & 64 & $(29.2)$ & 11 & $(36.7)$ & 75 & $(30.1)$ & \\
\hline Employed & 25 & $(27.8)$ & 21 & $(18.8)$ & 46 & $(22.8)$ & \multirow[t]{2}{*}{0.077} & 51 & (23.3) & 11 & $(36.7)$ & 62 & $(24.9)$ & \multirow[t]{2}{*}{0.080} \\
\hline Retired & 34 & $(37.8)$ & 60 & $(53.6)$ & 94 & $(46.5)$ & & 104 & $(47.5)$ & 8 & $(26.7)$ & 112 & $(45.0)$ & \\
\hline Religion: Islam & 79 & $(87.8)$ & 96 & $(85.7)$ & 175 & $(86.6)$ & 0.685 & 191 & $(87.2)$ & 30 & $(100)$ & 221 & $(88.8)$ & 0.032 \\
\hline \multicolumn{15}{|l|}{ Education } \\
\hline Elementary School & 13 & (14.4) & 8 & $(7.1)$ & 21 & $(10.4)$ & & 22 & $(10.0)$ & 8 & $(26.7)$ & 30 & $(12.0)$ & \\
\hline Junior High School & 8 & $(8.9)$ & 11 & $(9.8)$ & 19 & $(9.4)$ & \multirow{2}{*}{0.295} & 19 & $(8.7)$ & 5 & $(16.7)$ & 24 & (9.6) & \multirow{3}{*}{0.023} \\
\hline Senior High School & 28 & (31.1) & 31 & $(27.7)$ & 59 & $(29.2)$ & & 64 & $(29.2)$ & 6 & $(20.0)$ & 70 & $(28.1)$ & \\
\hline University & 41 & $(45.6)$ & 62 & $(55.4)$ & 103 & (51.0) & & 114 & (52.1) & 11 & (36.7) & 125 & $(50.2)$ & \\
\hline \multicolumn{15}{|l|}{ Smoking status } \\
\hline Never & 67 & $(74.4)$ & 72 & $(64.3)$ & 139 & (68.3) & \multirow{2}{*}{0.130} & 150 & (68.5) & 25 & (83.3) & 175 & $(70.3)$ & \multirow{2}{*}{0.068} \\
\hline Smoking & 23 & $(25.6)$ & 40 & $(35.7)$ & 63 & (31.2) & & 69 & (31.5) & 5 & $(16.7)$ & 74 & (29.7) & \\
\hline
\end{tabular}

DM Therapy

$\begin{array}{lcccccccccccccc}\text { Oral } & 29 & (32.2) & 45 & (40.2) & 74 & (36.6) & & 79 & (36.1) & 6 & (20.0) & 85 & (34.1) & \\ \text { Insulin } & 30 & (33.3) & 32 & (28.6) & 62 & (30.7) & & 68 & (31.1) & 16 & (53.3) & 84 & (33.7) & 0.020 \\ \text { Oral and Insulin } & 29 & (32.2) & 33 & (29.5) & 62 & (30.7) & & 6.692 & (31.1) & 6 & (20.0) & 74 & (29.7) & \\ \text { Nothing } & 2 & (2.2) & 2 & (1.8) & 4 & (2.0) & & 4 & (1.8) & 2 & (6.7) & 6 & (2.4)\end{array}$

Duration DM (years)†

\begin{tabular}{|c|c|c|c|c|c|c|c|c|c|c|c|c|c|c|}
\hline$<10$ & 49 & (54.4) & 57 & (50.9) & 106 & $(52.5)$ & & 114 & (52.1) & 18 & $(60.0)$ & 132 & $(53.0)$ & \\
\hline $11-20$ & 28 & (31.1) & 40 & (35.7) & 68 & (33.7) & \multirow{2}{*}{0.929} & 74 & (33.8) & 6 & $(20.0)$ & 80 & $(32.1)$ & \multirow{2}{*}{0.358} \\
\hline $21-30$ & 10 & $(11.1)$ & 11 & (9.8) & 21 & $(10.4)$ & & 23 & $(10.5)$ & 5 & $(16.7)$ & 28 & $(11.2)$ & \\
\hline$>30$ & 3 & (3.3) & 4 & (3.6) & 7 & (3.5) & & 8 & (3.7) & 1 & (3.3) & 9 & (3.6) & \\
\hline Mass Index $\left(\mathrm{Kg} / \mathrm{m}^{2}\right) \dagger$ & 26.1 & \pm 3.9 & 26.1 & \pm 4.0 & 26.1 & \pm 4.0 & 0.917 & 26.10 & \pm 4.0 & 26.08 & \pm 4.1 & 26.10 & \pm 4.0 & 0.974 \\
\hline \multicolumn{15}{|l|}{ Pressure $(\mathrm{mmHg}) \dagger$} \\
\hline Systolic & 133.9 & \pm 19.0 & 138.5 & \pm 20.1 & 136.5 & \pm 19.7 & 0.098 & 136.5 & \pm 19.3 & 134.0 & \pm 18.4 & 136.24 & \pm 19.1 & 0.496 \\
\hline Diastolic & 82.8 & \pm 8.8 & 82.8 & \pm 9.2 & 82.8 & \pm 9.0 & 0.980 & 83.0 & \pm 9.1 & 81.0 & \pm 10.2 & 82.77 & \pm 9.2 & 0.266 \\
\hline$(\%) \dagger$ & 8.07 & \pm 2.70 & 7.58 & \pm 2.42 & 7.80 & \pm 2.55 & 0.180 & 7.86 & \pm 2.61 & 8.95 & \pm 2.07 & 7.99 & \pm 2.57 & 0.029 \\
\hline IFCC $(\mathrm{mmol} / \mathrm{mol}) \dagger$ & 64.66 & \pm 29.56 & 59.36 & \pm 26.52 & 61.72 & \pm 27.97 & 0.181 & 62.38 & \pm 28.61 & 74.43 & \pm 22.66 & 63.83 & \pm 28.19 & 0.028 \\
\hline
\end{tabular}

*Excluded presence history DFU amputation. Data are $n(\%)$ unless indicated by $\dagger$ are mean $( \pm \mathrm{SD}) . p$ values determined by Chi Square $\mathrm{x}^{2}$ or Fisher exact test for categorical data and Independent $t$ test for continuous data. 
Table 2. Presence of Skin, nail and deformity problems for presence risk factors and diabetic foot ulcers (DFU).

\begin{tabular}{|c|c|c|c|c|c|c|c|c|c|c|c|c|c|c|}
\hline \multirow{2}{*}{ Skin Problems } & \multicolumn{6}{|c|}{ Foot at Risk } & \multicolumn{8}{|c|}{ Diabetic Foot Ulcer (DFU) } \\
\hline & \multicolumn{2}{|c|}{$\begin{array}{c}\text { No Risk } \\
\mathrm{n}=90(\%)\end{array}$} & \multicolumn{2}{|c|}{$\begin{array}{c}\text { Presence } \\
\text { Risk } \\
\text { n: } 112(\%)^{*}\end{array}$} & \multicolumn{2}{|c|}{$\begin{array}{c}\text { Total } \\
\text { n: } 202 \text { (\%) }\end{array}$} & \multirow{2}{*}{$\begin{array}{c}\mathrm{p} \\
0.261\end{array}$} & \multicolumn{2}{|c|}{$\begin{array}{c}\text { No DFU } \\
\text { n: } 219(\%)\end{array}$} & \multicolumn{2}{|c|}{$\begin{array}{c}\text { Presence DFU } \\
\text { n: } 30(\%)\end{array}$} & \multicolumn{2}{|c|}{$\begin{array}{c}\text { Total } \\
\text { n: } 249(\%)\end{array}$} & $\mathrm{p}$ \\
\hline Dry Skin & 12 & (13.3) & 22 & $(19.6)$ & 34 & $(16.8)$ & & 36 & $(16.4)$ & 13 & $(43.3)$ & 49 & (19.7) & 0.001 \\
\hline Callus, Corn, Fissure & 34 & $(37.8)$ & 45 & $(40.2)$ & 79 & (39.1) & 0.773 & 90 & $(41.1)$ & 16 & (53.3) & 106 & $(42.6)$ & 0.239 \\
\hline Tinea Pedis & 25 & $(27.8)$ & 34 & $(30.4)$ & 59 & $(29.2)$ & 0.756 & 63 & $(28.8)$ & 10 & (33.3) & 73 & (29.3) & 0.670 \\
\hline \multicolumn{15}{|l|}{ Nail Problems } \\
\hline Nail Deformity & 33 & $(36.7)$ & 44 & (39.3) & 77 & $(38.1)$ & 0.771 & 85 & $(38.8)$ & 17 & $(56.7)$ & 102 & $(41.0)$ & 0.075 \\
\hline Onychomycosis & 57 & $(63.3)$ & 76 & (67.9) & 133 & $(65.8)$ & 0.552 & 146 & $(66.7)$ & 24 & $(80.0)$ & 170 & $(68.3)$ & 0.151 \\
\hline Forefoot Deformity $\dagger$ & 0 & $(0.0)$ & 79 & $(70.5)$ & 79 & (39.1) & 0.000 & 87 & (39.7) & 15 & $(53.6)$ & 102 & $(41.3)$ & 0.221 \\
\hline Ankle Brachial Index $\ddagger$ & 1.04 & \pm 0.95 & 0.95 & \pm 0.14 & 0.99 & \pm 0.13 & 0.000 & 0.99 & \pm 0.13 & 0.97 & \pm 0.18 & 0.99 & \pm 0.13 & 0.529 \\
\hline Abnormal $(<0.9$ or $>1.3)$ & 0 & $(0)$ & 34 & $(30.4)$ & 34 & (16.8) & \multirow{2}{*}{0.000} & 36 & $(16.4)$ & 8 & (26.7) & 44 & $(17.7)$ & \multirow[b]{2}{*}{0.200} \\
\hline Normal (0.9 - 1.3) & 90 & $(100)$ & 78 & $(69.6)$ & 168 & $(83.2)$ & & 183 & $(83.6)$ & 22 & (73.3) & 205 & (82.3) & \\
\hline Toe Brachial Index & 0.47 & \pm 0.18 & 0.45 & \pm 0.15 & 0.46 & \pm 0.17 & 0.423 & 0.47 & \pm 0.17 & 0.41 & \pm 0.17 & 0.46 & \pm 0.17 & 0.081 \\
\hline
\end{tabular}

$p$ values determined by Chi Square $\mathrm{x}^{2}$ or Fisher exact test for categorical data and Independent $\mathrm{t}$ test for continuous data. ${ }^{*}$ Excluded presence history DFU amputation. ${ }^{\dagger}$ Forefoot Deformity including; hallux vagus, bunion, varsus deformity, hammer toe. ${ }^{\ddagger}$ ABI $>1.3$ were ignored and read only TBI data if presence.

daily foot inspection ( $P=0.003$ ), entirely foot inspection ( $\mathrm{P}=0.002)$, and supported for foot inspection by family $(\mathrm{P}=0.007)$. Related to the presence of DFU, daily five time foot washing or more $(\mathrm{P}=0.014)$, supported for foot washing by family $(P=0.021)$ and using shoes as footwear $(P=0.003)$ are associated with presence of DFU. In addition, cultural belief $(\mathrm{P}=0.036)$ and spiritual belief $(\mathrm{P}=0.007)$ related $\mathrm{DM}$ status has an evidence role in presence of risk and presence of DFU, respectively (Table 3).

\subsection{Multivariate Analysis}

Based on multicollinearity diagnostic we excluded supported for foot inspection (VIF 6.84) and daily foot washing (VIF 6.99). In addition by using logical approach we also excluded systolic blood pressure and occupation (not related to foot problems), forefoot deformity, ABI and TBI (used to distinguish risk category) and religion (100\% of DFU in group are Muslims).

Logistic regression was conducted to evaluate predictors of presence of risk and DFU. When all candidate predictors from univariate analysis entered together; age and daily foot inspection status were associated with presence risk, with an odds ratio (OR) 1.04 (95\% CI: 1.005 - 1.074) and 0.36 (95\% CI: 0.186 - 0.703), respectively. Meanwhile predictors for presence of DFU are DM therapy (insulin) OR 9.37 (95\% CI: 2.240 - 39.182), type of footwear (shoes) OR 0.05 (95\% CI: 0.007 - 0.294) and spiritual belief that DM was a disease OR 0.04 (95\% CI: 0.004 - 0.326) and belief that DM was temptation from God OR 0.13 (95\% CI: 0.027 - 0.598 ) (Table 4).

\section{Discussion}

\subsection{Prevalence of Risk and DFU}

Prevalence of risk and DFU are higher in Indonesia. The current study found that prevalence of risk (neuropathy and angiopathy) in this study was $55.4 \%$. These findings are within global prevalence of risk $40 \%-70 \%$ [21]. 
Table 3. Practical behavior related to foot care for presence risk factors and diabetic foot ulcers (DFU).

\begin{tabular}{|c|c|c|c|c|c|c|c|c|c|c|}
\hline \multirow[t]{2}{*}{ Practical Behaviors } & \multicolumn{4}{|c|}{ Foot at Risk } & \multicolumn{6}{|c|}{ Diabetic Foot Ulcer (DFU) } \\
\hline & \multicolumn{2}{|c|}{$\begin{array}{l}\text { No Risk } \\
\text { n: (\%) }\end{array}$} & \multicolumn{2}{|c|}{$\begin{array}{l}\text { Presence Risk } \\
\text { n: }(\%)^{*}\end{array}$} & $\mathrm{p}$ & \multicolumn{2}{|c|}{$\begin{array}{l}\text { No DFU } \\
\text { n (\%) }\end{array}$} & \multicolumn{2}{|c|}{$\begin{array}{c}\text { Presence DFU } \\
\mathrm{n}(\%)\end{array}$} & $\mathrm{p}$ \\
\hline Foot Inspection & \multicolumn{2}{|c|}{ n: 89} & \multicolumn{2}{|c|}{ n:107 } & & \multicolumn{2}{|c|}{$\mathrm{n}: 213$} & \multicolumn{2}{|c|}{$\mathrm{n}: 30$} & \\
\hline \multirow[t]{2}{*}{ Inspect foot daily } & 35 & (39.3) & 21 & (19.6) & 0.003 & 63 & (29.6) & 12 & $(40.0)$ & 0.292 \\
\hline & \multicolumn{2}{|c|}{ n: 87} & \multicolumn{2}{|c|}{ n: 110} & & \multicolumn{2}{|c|}{ n: 213} & \multicolumn{2}{|c|}{ n: 26} & \\
\hline \multirow[t]{2}{*}{ Inspect foot entirely } & 33 & (37.9) & 18 & $(16.4)$ & 0.002 & 56 & $(26.3)$ & 7 & $(26.9)$ & 0.952 \\
\hline & \multicolumn{2}{|c|}{ n: 87} & \multicolumn{2}{|c|}{ n: 110} & & \multicolumn{2}{|c|}{ n: 213} & \multicolumn{2}{|c|}{ n: 29} & \\
\hline Supported by family for foot inspection & 8 & $(9.2)$ & 1 & $(0.9)$ & 0.007 & 9 & $(4.2)$ & 4 & $(13.8)$ & 0.103 \\
\hline \multirow{3}{*}{$\begin{array}{l}\text { Foot Washing } \\
\text { Washing foot daily }\end{array}$} & \multicolumn{2}{|c|}{ n: 90} & \multicolumn{2}{|c|}{$\mathrm{n}: 112$} & & \multicolumn{2}{|c|}{ n: 29} & \multicolumn{2}{|c|}{$\mathrm{n}: 30$} & \\
\hline & 77 & $(85.6)$ & 100 & $(89.3)$ & 0.520 & 194 & (88.6) & 23 & (76.7) & 0.081 \\
\hline & & 89 & & & & & & & 29 & \\
\hline Five time or more foot washing & 69 & $(77.5)$ & 88 & $(79.3)$ & 0.714 & 173 & $(79.7)$ & 18 & $(62.1)$ & 0.014 \\
\hline & & 90 & & & & & & & 27 & \\
\hline Using moisturizer after foot washing & 9 & $(10.0)$ & 17 & $(15.2)$ & 0.473 & 26 & $(11.9)$ & 3 & $(11.1)$ & 1.000 \\
\hline & & & & & & & & & 30 & \\
\hline Supported by family for foot washing & 4 & $(4.5)$ & 5 & (4.5) & 0.697 & 11 & (5.1) & 6 & $(20.0)$ & 0.021 \\
\hline Nail Trim & & 90 & & & & & & & 30 & \\
\hline Weekly nail trim & 43 & $(47.8)$ & 49 & $(43.8)$ & 0.573 & 101 & $(46.1)$ & 17 & $(56.7)$ & 0.331 \\
\hline Nail trim tool & & & & & & & & & 29 & \\
\hline Knife, blade or scissor & 30 & (33.7) & 25 & $(22.3)$ & 0.081 & 59 & $(27.1)$ & 10 & $(34.5)$ & 0.509 \\
\hline Nail clipper or nail file & 59 & $(66.3)$ & 87 & $(77.7)$ & & 159 & $(72.9)$ & 19 & $(65.5)$ & \\
\hline & & & & & & & & & 29 & \\
\hline Supported by family for nail trim & 17 & (18.9) & 17 & $(15.2)$ & 0.571 & 41 & (18.8) & 9 & $(31.0)$ & 0.141 \\
\hline Foot Wear Inspection & & & & & & & & & 29 & \\
\hline Daily foot wear inspection & 28 & (31.1) & 28 & $(25.2)$ & 0.429 & 62 & (28.4) & 9 & $(31.0)$ & 0.828 \\
\hline & & & & & & & & & 29 & \\
\hline Inspect foot wear entirely & 26 & (28.9) & 24 & (21.8) & 0.526 & 54 & $(25.0)$ & 3 & $(10.3)$ & 0.196 \\
\hline & & & & & & & & & 28 & \\
\hline $\begin{array}{l}\text { Supported by family for foot wear } \\
\text { inspection }\end{array}$ & 4 & $(4.4)$ & 3 & $(2.7)$ & 0.760 & 9 & $(4.2)$ & 0 & $(0.0)$ & 0.713 \\
\hline Foot Wear Practice & & 90 & & & & & & & & \\
\hline Try foot wear before buy & 87 & $(96.7)$ & 102 & $(94.4)$ & 0.514 & 206 & $(95.8)$ & 26 & $(89.7)$ & 0.159 \\
\hline & & 89 & & & & & & & 30 & \\
\hline Measure foot wear before buy & 64 & (71.9) & 69 & $(62.2)$ & 0.175 & 147 & (68.1) & 8 & (60) & 0.410 \\
\hline
\end{tabular}




\section{Continued}

\begin{tabular}{|c|c|c|c|c|c|c|c|c|c|c|}
\hline \multirow[b]{2}{*}{ Using foot wear inside home } & \multicolumn{2}{|c|}{ n: 90} & \multicolumn{2}{|c|}{ n: 109} & \multirow[b]{2}{*}{0.880} & \multicolumn{2}{|c|}{ n: 216} & \multicolumn{2}{|c|}{ n: 30} & \multirow[b]{2}{*}{0.684} \\
\hline & 30 & (33.3) & 35 & $(32.1)$ & & 75 & $(34.7)$ & 2 & $(40.0)$ & \\
\hline \multirow{3}{*}{ Using foot wear outside home } & \multicolumn{2}{|c|}{ n: 90} & \multicolumn{2}{|c|}{ n: 111} & & \multicolumn{2}{|c|}{ n: 218} & \multicolumn{2}{|c|}{ n: 30} & \\
\hline & 85 & $(94.4)$ & 102 & $(91.9)$ & 0.583 & 203 & $(93.1)$ & 6 & $(86.7)$ & 0.262 \\
\hline & \multicolumn{2}{|c|}{ n: 90} & \multicolumn{2}{|c|}{ n: 111} & & \multicolumn{2}{|c|}{ n: 218} & \multicolumn{2}{|c|}{ n: 29} & \\
\hline Using shoes as foot wear & 37 & (41.1) & 41 & (36.9) & 0.563 & 84 & $(38.5)$ & 3 & (10.3) & 0.003 \\
\hline Cultural belief related to DM status & \multicolumn{2}{|c|}{ n: 90} & \multicolumn{2}{|c|}{ n: 112} & & \multicolumn{2}{|c|}{ n: 219} & \multicolumn{2}{|c|}{$\mathrm{n}: 30$} & \\
\hline Nothing & 60 & $(66.7)$ & 90 & $(80.4)$ & & 163 & $(74.4)$ & 21 & $(70)$ & \\
\hline DM can be avoided by cultural approach & 5 & $(5.6)$ & 1 & $(0.9)$ & 0.036 & 8 & (3.7) & 1 & (3.3) & 0.858 \\
\hline DM is just about food problems & 25 & $(27.8)$ & 21 & $(18.8)$ & & 48 & $(21.9)$ & 8 & $(26.7)$ & \\
\hline Spritual belief related to DM status & \multicolumn{2}{|c|}{ n: 90} & \multicolumn{2}{|c|}{ n: 112} & & \multicolumn{2}{|c|}{ n: 219} & \multicolumn{2}{|c|}{ n: 30} & \\
\hline Nothing & 5 & (5.6) & 6 & (5.4) & & 12 & (5.5) & 5 & (16.7) & \\
\hline $\mathrm{DM}$ is just a disease & 26 & (28.9) & 36 & (32.1) & & 64 & $(29.2)$ & 2 & (6.7) & \\
\hline DM is temptation from God & 55 & $(61.1)$ & 61 & $(54.5)$ & 0.692 & 129 & (58.9) & 20 & $(66.7)$ & 0.007 \\
\hline Others & 4 & (4.4) & 9 & $(8.0)$ & & 14 & (6.4) & 3 & (10) & \\
\hline
\end{tabular}

$p$ values determined by Chi Square $\mathrm{x}^{2}$ or Fisher exact test for categorical data and Independent t test for continuous data. ${ }^{*}$ Excluded presence history DFU amputation.

Table 4. Multivariate logistic regression analysis of predictors for presence risk factors and diabetic foot ulcers (DFU).

\begin{tabular}{|c|c|c|}
\hline Predictors & \multicolumn{2}{|c|}{ OR (95\% CI). } \\
\hline \multicolumn{3}{|l|}{ Predictors For Presence Risk } \\
\hline Age & 1.04 & $(1.005-1.074)$ \\
\hline \multicolumn{3}{|l|}{ Daily foot inspection } \\
\hline No & \multicolumn{2}{|c|}{ Reference } \\
\hline Yes & 0.36 & $(0.186-0.703)$ \\
\hline \multicolumn{3}{|l|}{ Predictors For Presence DFU } \\
\hline \multicolumn{3}{|l|}{ DM therapy } \\
\hline Oral & \multicolumn{2}{|c|}{ Reference } \\
\hline Insulin & 9.37 & $(2.240-39.182)$ \\
\hline Oral or Insulin & 2.38 & $(0.507-11.199)$ \\
\hline Nothing & 1.99 & $(0.077-51.267)$ \\
\hline \multicolumn{3}{|l|}{ Type of foot wear } \\
\hline Barefoot or Sandal & \multicolumn{2}{|c|}{ Reference } \\
\hline Shoes & 0.05 & $(0.007-0.294)$ \\
\hline \multicolumn{3}{|l|}{ Spiritual belief related to DM status } \\
\hline No Spiritual belief & \multicolumn{2}{|c|}{ Reference } \\
\hline DM was a disease & 0.04 & $(0.004-0.326)$ \\
\hline DM was a temptation from God & 0.13 & $(0.027-0.598)$ \\
\hline Others spiritual belief & 0.24 & $(0.028-2.157)$ \\
\hline
\end{tabular}


This percentage still remains higher compared to India [22]. Meanwhile, current prevalence of DFU is $12 \%$. These findings are higher compared to China (most populated DM country) [23] and in comparison with global prevalence $1.4 \%$ - 5.9\% [21]. In addition, our previous study also confirmed high prevalence of DFU in home care setting 26.0\% [24]. Prevalence of risk and DFU in Indonesia is potential to be higher since the prevalence of undiagnosed DM in Indonesia was higher [4] [5], including prediction of prevalence DM in Indonesia [1] [2]. Thus, the findings indicate the importance of preventive strategies to prevent risk and DFU in Indonesia.

\subsection{Predictors for Presence of Risk}

There are two predictors for presence of risk, age and daily foot inspection. Current study also finds that age is associated with presence of risk. These results are consistent with previous study that increasing age linear with increasing risk for neuropathy and angiopathy [25]. Regarding daily foot inspection status (OR: 0.36), participants who perform daily foot inspection were less prevalent to presence of risk factors. The findings indicate the importance of foot inspection to prevent presence of risk factors.

\subsection{Predictors for DFU}

The strengths of this study are, besides insulin therapy, mainly predictors for presence of DFU related to foot care behaviors (using shoes as footwear and spiritual belief that DM was a disease or temptation from God). These predictors are preventable by increasing knowledge related to foot care.

Current study revealed that participants who used insulin were associated with presence DFU, similar with Europe [26] and in Asia study [23]. This finding might be explained by the fact that DM therapy reflect severity of glycemic status which increase risk for DFU. In addition, $\mathrm{HbA}_{1 \mathrm{C}}$ was associated with presence DFU in univariate analysis, but diminished in multivariate analysis as well as others studies [26].

Another important result is that the spiritual belief is associated with presence of DFU. As we know, the role of spiritual belief against DFU has not been explained in previous studies. Current study indicates that spiritual belief of DM is a disease or temptation from God has less likely to presence of DFU compared to who has no spiritual belief. One of the potential answers is that the spiritual belief might give results to positive behaviors related to foot care. Previous study confirmed that spiritual belief associated with positive coping among T2DM patients [27] and has a role in controlling glycemic status [28].

Another predictor is related to footwear practice. In this study, using shoes as footwear is less likely to presence of DFU compared to who use sandals. Most of the Indonesian people use sandals as footwear which only covers the plantar of foot area, as a result the foot remains at high risk from external trauma. Even though effectiveness of footwear to prevent DFU remains unclear [29], these findings emphasize the important effect of shoes as footwear to prevent DFU compared sandals.

\section{Limitations}

We note there were three limitations in this study. First, the sample size was relatively small and failed to reach a calculated sample size since the last month of study that there was no more additional attendance. Secondly, research setting was conducted only in one hospital, which partially reflected clinical problems in Indonesia. In addition, since the setting was a regional hospital in eastern Indonesia, which received referrals from other hospitals, the attendance participants were at high risk status. As a result half of participants were at risk group. Last, limitation was related to research design. Research design of current study is a cross sectional epidemiological study. Consequently, the result of this study indicated about relationship data for presence of risk and presence of DFU rather that causal-effect. Thus, further multisite prospective study can be represented to evaluate prevalence of DFU in Indonesia.

\section{Conclusions}

As we know, this is the first epidemiological study in Indonesia which investigates prevalence, associated risk factors and predictors of DFU. Even though the prevalence of DFU is high, identification associated factors for presence of risk and DFU has not been integrated into national guideline [30]. Thus, preventive strategies should be introduced at early stage to prevent presence of risk and DFU.

Interestingly, we note among all predictors (for presence of risk and DFU) can be modified by into positive 
behaviors. So far, many studies are related to the associated factor for presence of risk and DFU has focused on physiological problems (neuropathy and angiopathy) and seem to ignore patients' behaviors related to their illness status. In fact, previous study has revealed that the importance of patients' belief is determinant for foot care [31]. Thus, modified belief on behaviors is more important rather than belief of illness [32]. In conclusion, we recommend to educate high risk patients to understand positive foot care behavior as essential preventive strategies to prevent presence of risk and DFU.

\section{References}

[1] Shaw, J.E., Sicree, R.A. and Zimmet, P.Z. (2010) Global Estimates of the Prevalence of diabetes for 2010 and 2030. Diabetes Research and Clinical Practice, 87, 4-14. http://dx.doi.org/10.1016/j.diabres.2009.10.007

[2] Guariguata, L., Whiting, D.R., Hambleton, I., Beagley, J., Linnenkamp, U. and Shaw, J.E. (2014) Global Estimates of Diabetes Prevalence for 2013 and Projections for 2035. Diabetes Research and Clinical Practice, 103, 137-149. http://dx.doi.org/10.1016/j.diabres.2013.11.002

[3] Waspadji, S., Ranakusuma, A., Suyono, S., Supartondo, S. and Sukaton, U. (1983) Diabetes Mellitus in an Urban Population in Jakarta, Indonesia. The Tohoku Journal of Experimental Medicine, 141, 219-228. http://dx.doi.org/10.1620/tjem.141.Suppl_219

[4] Mihardja, L., Delima, Siswoyo, H., Ghani, L. and Soegondo, S. (2009) Prevalence and Determinants of Diabetes Mellitus and Impaired Glucose Tolerance in Indonesia (a Part of Basic Health Research/Riskesdas). Acta Medica Indonesiana, 41, 169-74.

[5] Soewondo, P., Ferrario, A. and Tahapary, D.L. (2013) Challenges in Diabetes Management in Indonesia: A Literature Review. Global Health, 9, 1-17. http://dx.doi.org/10.1186/1744-8603-9-63

[6] International Working Group on the Diabetic Foot (IWGDF) (2013) Pathophysiology of Foot Ulceration. http://iwgdf.org/consensus/pathophysiology-of-foot-ulceration/

[7] Fernando, M., Crowther, R., Lazzarini, P., et al. (2013) Biomechanical Characteristics of Peripheral Diabetic Neuropathy: A Systematic Review and Meta-Analysis of Findings from the Gait Cycle, Muscle Activity and Dynamic Barefoot Plantar Pressure. Clinical Biomechanics, 28, 831-845. http://dx.doi.org/10.1016/j.clinbiomech.2013.08.004

[8] Huijeberts, M.S.P., Schaper, N.C. and Schalkwijk, C.G. (2007) Advanced Glycation end Products and Diabetic Foot Disease. Diabetes/Metaolism Research and Reviews, 24, 19-24. http://dx.doi.org/10.1002/dmrr.861

[9] Malgrange, D., Richard, J.L. and Leymarie, F. (2003) Screening Diabetic Patients at Risk for Foot Ulceration. A MultiCentre Hospital-Based Study in France. Diabetes and Metabolism, 29, 261-268. http://dx.doi.org/10.1016/S1262-3636(07)70035-6

[10] Reiber, G.E., Vileikyte, L., Boyko, E.J., Smith, D.G., Lavery, L.A. and Boulton, A.J.M. (1999) Causal Pathways for Incident Lower-Extremity Ulcers in Patients with Diabetes from Two Setting. Diabetes Care, 22, 157-162. http://dx.doi.org/10.2337/diacare.22.1.157

[11] Vandenbroucke, J.P., Von Elm, E., Altman, D.G., Gotzsche, P.C., Mulrow, C.D., Pocock, S.J., et al. (2007) Strengthening the Reporting of Observational Studies in Epidemiology (STROBE): Explanation and Elaboration. Epidemiology, 18, 805-835. http://dx.doi.org/10.1097/EDE.0b013e3181577511

[12] Sutanegara, D. and Budhiarta, D.A. (2000) The Epidemiology and Management of Diabetes Mellitus in Indonesia. Diabetes Research and Clinical Practice, 50, S9-S16. http://dx.doi.org/10.1016/s0168-8227(00)00173-x

[13] Adam, F.M.S., Adam, J.M.F., Pandeleki, N. and Mappangara, I. (2000) Asymptomatic Diabetes: The Difference between Population-Based and Office-Based Screening. Acta Medica Indonesiana, 38, 67-71.

[14] Hu, L., Bao, X., Zhou, S., Guan, X. and Xin, H.L. (2011) Estimation of Sample Size and Testing Power (Part 1). Journal of Integrative Medicine, 9, 1070-1074.

[15] Boulton, A.J.M., Armstrong, D.G., Albert, S.F., Frykberg, R.G., Hellman, R., Kirkman, M.S., et al. (2008) Comprehensive Foot Examination and Risk Assessment: A Report of the Task Force of the Foot Care Interest Group of the American Diabetes Association, with Endorsement by the American Association of Clinical Endocrinologists. Diabetes Care, 31, 1679-1685. http://dx.doi.org/10.2337/dc08-9021

[16] American Diabetes Association (ADA) (2013) Executive Summary: Standards of Medical Care in Diabetes 2013. Diabetes Care, 36, S4-S10.

[17] Tan, L.S. (2010) The Clinical Use of the 10g Monofilament and Its Limitations: A Review. Diabetes Research and Clinical Practice, 90, 1-7. http://dx.doi.org/10.1016/j.diabres.2010.06.021

[18] Aboyans, V., Criqui, M.H., Abraham, P., Allison, M.A., Creager, M.A., Diehm, C., et al. (2012) Measurement and Interpretation of the Ankle-Brachial Index: A Scientific Statement from the American Heart Association. Circulation, 126, 2890-2909. http://dx.doi.org/10.1161/CIR.0b013e318276fbcb 
[19] Høyer, C., Sandermann, J. and Petersen, L.J. (2013) The Toe-Brachial Index in the Diagnosis of Peripheral Arterial Disease. Journal of Vascular Surgery, 58, 231-238. http://dx.doi.org/10.1016/j.jvs.2013.03.044

[20] Schaper, N.C. (2004) Diabetic Foot Ulcer Classification System for Research Purposes: A Progress Report on Criteria for Including Patients in Research Studies. Diabetes/Metabolisme Research and Review, 20, 90-95. http://dx.doi.org/10.1002/dmrr.464

[21] Boulton, A.J.M. (2008) The Diabetic Foot: Grand Overview, Epidemiology and Pathogenesis. Diabetes/Metabolisme Research and Review, 24, S3-S6. http://dx.doi.org/10.1002/dmrr.833

[22] Bansal, D., Gudala, K., Muthyala, H., Esam, H.P., Nayakallu, R. and Bhansali, A. (2014) Prevalence and Risk Factors of Development of Peripheral Diabetic Neuropathy in Type 2 Diabetes Mellitus in a Tertiary Care Setting. Journal of Diabetes Investigation, 5, 714-721. http://dx.doi.org/10.1111/jdi.12223

[23] Jiang, Y., Wang, X., Xia, L., Fu, X., Xu, Z., Ran, X., et al. (2015) A Cohort Study of Diabetic Patients and Diabetic Foot Ulceration Patients in China. Wound Repair and Regeneration, 23, 222-230. http://dx.doi.org/10.1111/wrr.12263

[24] Yusuf, S., Kasim, S., Okuwa, M. and Sugama, J. (2013) Development of an Enterostomal Therapy Nurse Outpatient Wound Clinic in Indonesia: A Retrospective Descriptive Study. Wound Practice and Research, 21, 41-47.

[25] Gregg, E.W., Sorlie, P., Paulose-Ram, R., Gu, Q., Eberhardt, M.S., Wolz, M., et al. (2004) Prevalence of Lower-Extremity Disease in the US Adult Population $\geq 40$ Years of Age with and without Diabetes. Diabetes Care, 27, 15911597. http://dx.doi.org/10.2337/diacare.27.7.1591

[26] Boyko, E.J., Ahroni, J.H., Stensel, V., Forsberg, R.C., Davignon, D.R. and Smith, D.G. (1999) A Prospective Study of Risk Factors for Diabetic Foot Ulcer. Diabetes Care, 22, 1036-1042. http://dx.doi.org/10.2337/diacare.22.7.1036

[27] Lundberg, P.C. and Thrakul, S. (2013) Religion and Self-Management of Thai Buddhist and Muslim Women with Type 2 Diabetes. Journal of Clinical Nursing, 22, 1907-1916. http://dx.doi.org/10.1111/jocn.12130

[28] Newlin, K., Melkus, G., Tappen, R., Chyun, D. and Koenig, H. (2008) Relationships of Religion and Spirituality to Glycemic Control in Black Women with Type 2 Diabetes. Nursing Research, 57, 331-339. http://dx.doi.org/10.1097/01.NNR.0000313497.10154.66

[29] Healy, A., Naemi, R. and Chockalingam, N. (2013) The Effectiveness of Footwear as an Intervention to Prevent or to Reduce Biomechanical Risk Factors Associated with Diabetic Foot Ulceration: A Systematic Review. Journal of Diabetes and Its Complications, 27, 391-400. http://dx.doi.org/10.1016/j.jdiacomp.2013.03.001

[30] Widyahening, I.S., Van der Graaf, Y, Soewondo, P., Glasziou, P. and Van der Heijden, G.J.M.G. (2014) Awareness, Agreement, Adoption and Adherence to Type 2 Diabetes Mellitus Guidelines: A Survey of Indonesian Primary Care Physicians. BMC Family Practice, 15, 72. http://dx.doi.org/10.1186/1471-2296-15-72

[31] Vedhara, K., Dawe, K., Wetherell, M.A., Miles, J.N., Cullum, N., Dayan, C., et al. (2014) Illness Beliefs Predict SelfCare Behaviours in Patients with Diabetic Foot Ulcers: A Prospective Study. Diabetes Research and Clinical Practice, 106, 67-72. http://dx.doi.org/10.1016/j.diabres.2014.07.018

[32] French, D.P., Wade, A.N. and Farmer, A.J. (2013) Predicting Self-Care Behaviours of Patients with Type 2 Diabetes: The Importance of Beliefs about Behaviour, Not Just Beliefs about Illness. Journal of Psychosomatic Research, 74, 327-333. http://dx.doi.org/10.1016/j.jpsychores.2012.12.008 